\title{
Multisession CyberKnife radiosurgery for optic nerve sheath meningiomas
}

\author{
Pantaleo Romanelli, M.D., ${ }^{1}$ Berndt Wowra, M.D., ${ }^{2}$ and Alexander Muacevic, M.D. ${ }^{2}$ \\ ${ }^{1}$ Functional Neurosurgery, IRCCS Neuromed, Pozzilli, Italy; and ${ }^{2}$ European CyberKnife Center, \\ Munich, Germany
}

\begin{abstract}
$\checkmark$ Optic nerve sheath meningiomas (ONSMs) are benign lesions originating from the dural sheath of the optic nerve. Progressive growth can lead to gradual loss of vision and exophthalmos. Loss of vision following microsurgical resection is not uncommon, and although stereotactic fractionated radiotherapy can be a safe alternative to control tumor growth and preserve vision, it may also lead to complications. Frame-based stereotactic radiosurgery has only been rarely used because single-fraction high-dose treatments of intrinsic optic nerve lesions may induce unacceptably high toxicity. New frameless radiosurgery devices such as the robotic CyberKnife, an image-guided radiosurgery system, can provide the extremely tight conformality and submillimetric accuracy of frame-based systems combined with the possibility of delivering radiation in several sessions. In the present report the authors review the clinical presentation and management of ONSMs and describe their preliminary experience using multisession radiosurgery to treat these lesions. (DOI: 10.3171/FOC-07/12/E11)
\end{abstract}

\section{KEY WoRdS • CyberKnife • image guidance • optic nerve sheath meningioma • robotics - stereotactic radiosurgery}

$\mathrm{P}$ RIMARY orbital meningiomas arising from the optic nerve dural sheath are called ONSMs. They typically grow by spreading circumferentially along the optic nerve, leading to nerve atrophy due to compression and failure of the pial blood supply. They can be located on the orbital or canalicular portion of the optic nerve. The latter type is typically detected early when it causes compression within the optic canal, but the former may grow large while visual deficits progress slowly. Bilateral visual deficits may be caused by direct extension or by traction and distortion of the optic chiasm (Fig. 1).

The most common symptoms of ONSMs are loss of vision and exophthalmos. ${ }^{2,5}$ In particular, progressive loss of visual acuity or visual fields is present in nearly all the cases examined, even when the lesion is small. The most common visual field defect is peripheral constriction. Early stages of compressive neuropathy usually manifest as dyschromatopsia, afferent pupillary defects, and optic disc edema, which may lead to optic atrophy. Opticociliary shunt vessels are a late sign of compressive optic neuropathy and are often associated with exophthalmos. The triad of progressive painless visual loss, optic nerve atrophy, and optic-

Abbreviations used in this paper: $\mathrm{CT}=$ computed tomography; LINAC = linear accelerator; MR = magnetic resonance; ONSM = optic nerve sheath meningioma; SFRT $=$ stereotactic fractionated radiotherapy. ociliary shunt vessels is rare but virtually pathognomonic for the diagnosis of ONSM.

\section{Clinical Management of ONSMs}

Because ONSMs and the symptoms they cause progress slowly, conservative management has often been advocated; treatment may be deferred until evidence of progression is found. This "wait and see" approach is reinforced by the high likelihood of vision loss after microsurgical resection. Conventional radiotherapy is more likely to preserve vision but is less likely than surgery to produce local tumor growth control. Radiotherapy is also associated with a risk of radionecrosis and radiation-induced neuropathy of cranial nerves.

This conservative approach, which has dominated the field over the last 60 years, can be challenged based on good results recently reported using stereotactic techniques of radiation delivery. Growing evidence supports the use of fractionated stereotactic radiation therapy in the management of symptomatic ONSMs. Single-session (Gamma Knife or LINAC-based) radiosurgery has rarely been offered as treatment for ONSMs because it is feared that effective treatment doses would not be tolerated by the optic nerve. Recent reports suggest, however, that effective single-session radiosurgery may be tolerated (J Adler, personal communication). ${ }^{10,11}$ Recent technical innovations have eliminated the stereotactic frame that has generally been 
used for single-session radiosurgery, thereby making multisession radiosurgery a feasible alternative. Delivering high radiation doses stereotactically in multiple sessions may provide ONSM growth control in association with increased probability of sparing vision. Preliminary experience treating ONSMs with multisession CyberKnife radiosurgery, which we review below, appears quite promising.

\section{Microsurgical Treatment}

Encasement of the optic nerve by an ONSM (Fig. 1) makes it impossible to obtain a radical resection without causing severe injury to the nerve itself or to its vascular supply, which is completely shared by the tumor. As a consequence, vision preservation following microsurgical resection of ONSMs is rare. ${ }^{2,7}$ Some experienced surgeons believe that excision invariably leads to blindness. ${ }^{9}$ As a general attitude toward the most common type of ONSMs, a fusiform sheath growing around the optic nerve, most surgeons do not even attempt to dissect the nerve but directly proceed to the resection of the nerve and the tumor together. Nerve preservation in these cases is clearly impossible.

Optic nerve sheath meningiomas confined in the orbit are resected via an orbitotomy.5,9 Orbital exenteration is performed when the orbit is invaded by tumor beyond the optic nerve. ${ }^{2}$ For meningiomas extending into the optic canal from within the skull, surgical unroofing is the preferred treatment. The surgical approach in this situation depends on the location of the meningioma in relation to the optic nerve. ${ }^{24}$ Craniotomy is required in cases of intracranial extension. En bloc resection of the optic nerve is likely to be the best strategy in patients in whom functional vision is lost, especially if there is extensive intracranial extension. Surgery is important also in patients with disfiguring exophthalmos and blindness. Young patients with good vision may harbor meningiomas that behave more aggressively and thus carry a poor prognosis. In these cases the risk of chiasmal involvement and bilateral loss of vision should be contemplated and early surgery may be attempted.

\section{Stereotactic Fractionated Radiation Therapy}

Stereotactic fractionated radiation and conformal radiotherapy have been used increasingly to treat ONSMs. Favorable short-term results have been published, and primary SFRT has been advocated to preserve vision in patients with ONSM rather than undertaking observation alone. ${ }^{3}$ Some authors assume that SFRT will become a standard treatment approach for ONSM. . $^{3,10,13,15,17,18,21,29}$ Stereotactic fractionated radiotherapy produces conformal dose distributions with consequent substantial sparing of surrounding normal tissues and is clearly a more sophisticated and safer treatment option than conventional radiotherapy. On the other hand, it does not reach the extremely tight dose distribution typical of radiosurgery and may still allow dose leaking over the retina and other nearby structures. As a consequence, radiation retinopathy, loss of vision, and endocrine failure may still occur after SFRT for ONSMs. ${ }^{4,27}$ Radiation optic neuropathy has also been described after low-dose treatment (40-45 Gy delivered at $<2$ Gy/fraction) of pituitary lesions. ${ }^{30}$ Furthermore, sever-

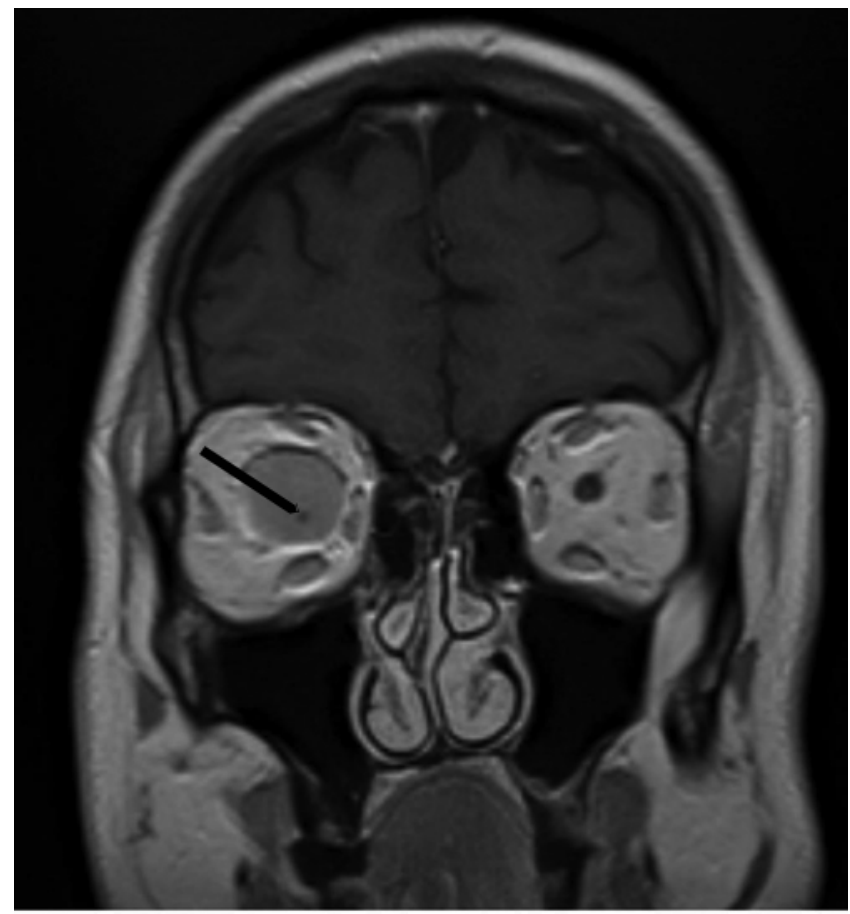

FIG. 1. Case 2. Coronal T1-weighted contrast-enhanced MR image of the orbit. The black arrow shows an atrophic optic nerve eccentrically placed inside the globular contrast-enhancing meningioma. Preservation of a functional nerve through microsurgical resection is beyond the current limits of the technique.

al other long-term ophthalmic and adnexal complications following external-beam radiotherapy and SFRT have been described ${ }^{8}$

\section{Frameless Image-Guided Robotic Radiosurgery}

\section{Technological Overview}

The CyberKnife is a frameless 6 MV LINAC system for image-guided robotic radiosurgery (Fig. 2). Real-time image guidance based on digitally reconstructed skull radiographs is used to localize and treat the targeted site. The robotic arm provides great flexibility and the highest number of noncoplanar penetration trajectories ( $\leq 1600$ with the new G4 model). Radiation is delivered with submillimetric accuracy. The system presumes a fixed relationship between the target and the skull, as with other forms of stereotaxy. A light-weight 6-MV LINAC is accurately positioned by a robotic arm with 6 degrees of freedom. Two x-ray imaging devices positioned on either side of the patient's anatomy acquire real-time digital radiographs of the skull at repeated intervals during treatment. Amorphous silicon sensors create a high-quality image of the skull or spine with a modest exposure to radiation $(10 \mathrm{~mA}, 75 \mathrm{kV}$; corresponding to a dose per image of $\sim 25 \mathrm{mrad}$ ).

The images acquired during the treatment are automatically registered to digitally reconstructed radiographs derived from the treatment planning CT scans. This registration process allows the position of the skull (and thus the 


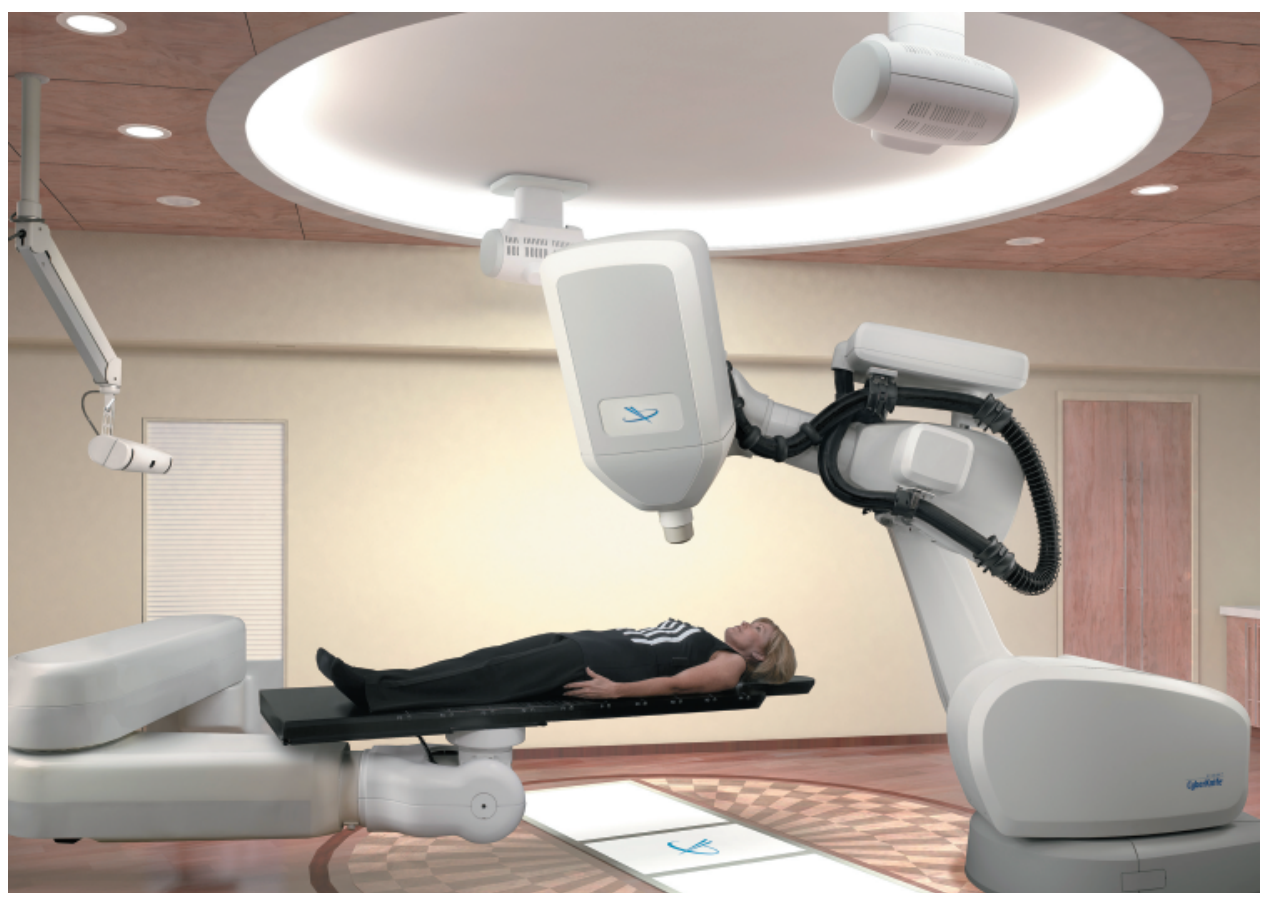

FIG. 2. The CyberKnife setup. A lightweight LINAC is mounted on a robotic arm and guided by real-time 6D digital radiographic imaging provided by amorphous silicon detectors.

treatment site) to be translated to the coordinate frame of the LINAC. A control loop between the imaging system and the robotic arm adjusts the pointing of the LINAC therapeutic beam to the observed position of the treatment anatomy (target). If the patient moves, the change is detected during the next imaging cycle and the beam is adjusted and realigned

\section{CyberKnife Treatment of Optic Pathway Lesions}

The CyberKnife offers 2 distinct advantages in the treatment of optic pathway lesions, both stemming from the absence of a rigid frame. Real-time image guidance based on digital $6 \mathrm{D}$ reconstruction of radiographic images of the skull provides accurate beam delivery for either single- or multisession treatments. By delivering treatment in multiple sessions it is possible to exploit the differential speed of recovery of normal and pathological tissues, thereby limiting or avoiding damage to the visual pathways while controlling tumor growth. ${ }^{1,6,20}$ Furthermore, many more trajectories are available due to the absence of a rigid frame blocking the access of beams through the basicranium and the facial bones (Fig. 3). These additional beam penetration trajectories, which are not available in the presence of a rigid frame attached at the level of the orbitomeatal line, provide enhanced dose conformality and homogeneity while greatly reducing the number of beams crossing the brain. Inverse planning software is used to optimize target coverage and achieve tightly conformal isodose distributions with substantial sparing of surrounding brain tissue (Fig. 3).22,23 Nonisocentric beam delivery (as opposed to isocentric multishot technology) also enables a significant measure of dose homogeneity.

Multisession treatment protocols involving the CyberKnife to treat optic pathway lesions have been reported to result in excellent rates of tumor control or arteriovenous malformation obliteration and good preservation of vision. ${ }^{1,20,25}$

\section{Multisession CyberKnife Radiosurgery for ONSMS}

Radiosurgical treatment of anterior visual pathway lesions is an especially challenging task due to the peculiar radiation sensitivity of the optic nerve chiasm and tracts. On the basis of the preliminary experience in the treatment of optic pathway lesions reported by the Stanford CyberKnife team led by John Adler, multisession CyberKnife radiosurgery was offered to 3 of our patients with ONSMs. The diagnosis was based on neuroimaging; no biopsy sample was obtained. The tumor originated from the orbital segment of the optic nerve in 2 patients and from the canalicular segment in 1 . Presenting symptoms included visual field deficits, loss of visual acuity, and exophthalmos associated with opticociliary shunt vessels (Table 1). A slow but appreciable worsening of visual complaints occurred in all 3 patients in the absence of a clear growth of the lesion on serial neuroimaging. However, surgery was deferred due to the presence of residual visual function, and the patients were offered the option of undergoing multisession CyberKnife treatment based on the good results in treating visual pathway lesions following the Stanford multisession protocol. The patients underwent thin-slice (1.25mm-thick) CT scanning after intravenous injection of contrast medium, as well as volumetric MR imaging. The studies were fused and the targets identified. In all 3 cases, side-by-side comparison of the studies showed that the contrast-enhanced CT scans were a sufficient and satisfactory study to identify the nerve and the tumor. It was therefore possible to draw the target directly on the CT scans, which probably increased substantially the accuracy of the 


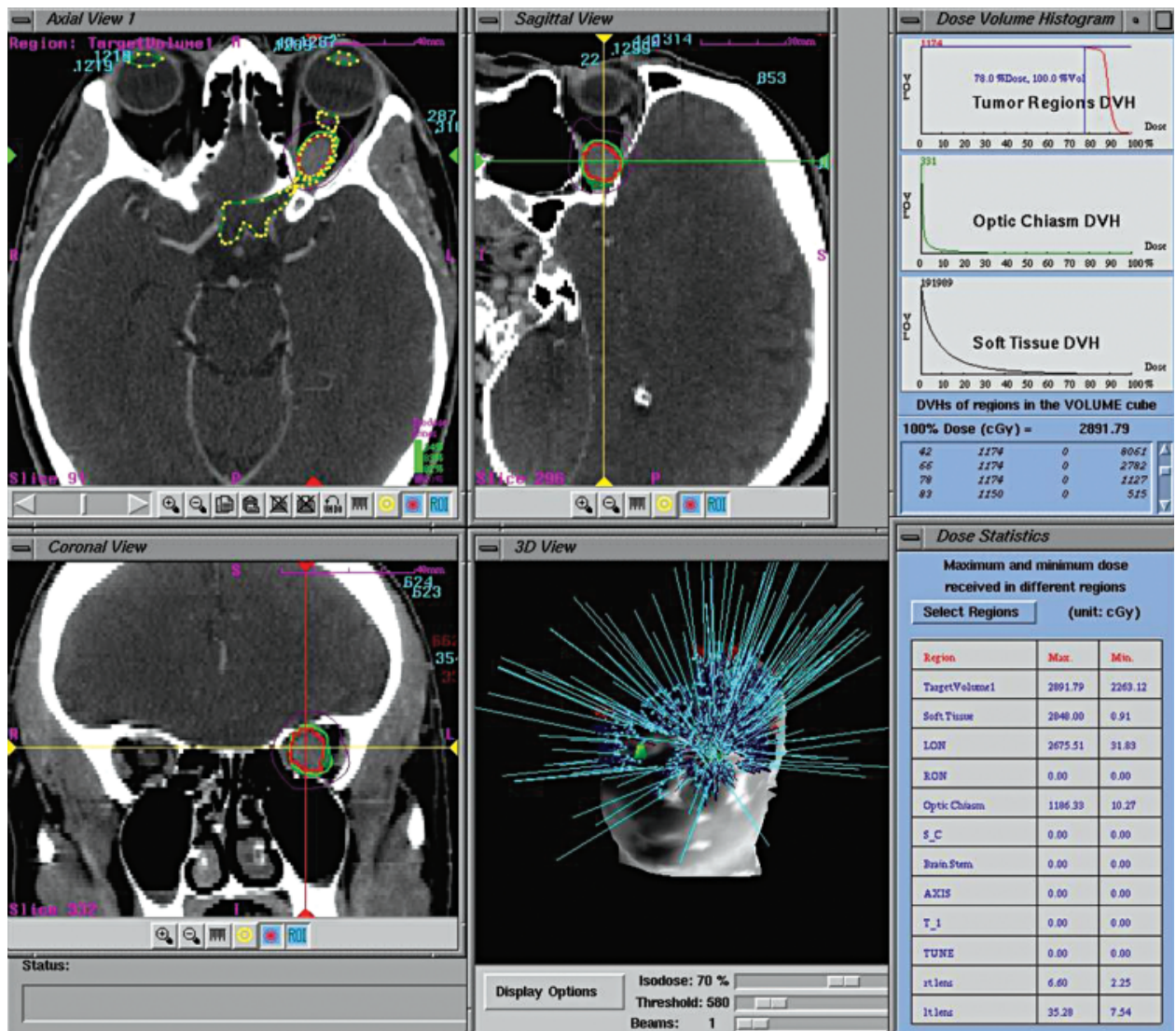

FIG. 3. CyberKnife treatment planning screenshot of a left ONSM, showing axial, sagittal, and coronal images as well as the maximum dose $(28.9 \mathrm{~Gy})$, collimator size $(10 \mathrm{~mm})$ and dose-volume histograms. The green line is the prescribed isodose $(80 \%)$, and the purple line is the $50 \%$ isodose. The image in the lower right panel depicts the multiple beam trajectories proposed by the treatment planning system. It can be immediately appreciated by the vast number of beams penetrating the face and the basicranium with consequent sparing of brain irradiation. $\mathrm{DVH}=$ dose-volume histogram.

treatment because the error introduced by the distortion in MR studies was avoided, as was the further error induced by CT-MR image fusion. The optic nerve was found to be embedded in the tumor in 1 case (Case 2 in Table 1; also see Fig. 1), whereas in the other 2 cases the optic nerve appeared as a marginal structure compressed and displaced by the tumor. In the latter 2 cases the nerve was drawn out as a critical structure during treatment planning and, despite the proximity to the tumor, a treatment plan that minimized the dose delivered to the optic nerve was created. Care was taken to limit the maximum dose to $<30 \mathrm{~Gy}$ and to obtain a tightly conformal and homogeneous dose distribution. Particular care was also taken to reduce to a minimum the radiation dose to the lens, retina, and optic nerve beyond the margins of the tumor. An $80 \%$ prescribed isodose of 20 Gy was delivered in 4 sessions of 5 Gy, each lasting 25 minutes and separated by a 24-hour interval. Dexamethasone (4 mg) was administered after each session. No further steroid agents were required during follow-up.

Follow-up consisted of MR imaging (every 6 months) and visual field and acuity examinations (every 3 months). No changes in lesion size were observed over time on serial images. Progressive improvement in visual fields and acuity was documented in all 3 patients. After 1 year, full restoration to normal vision was diagnosed by independent ophthalmologists assessing these patients. Visual fields and acuity restoration have remained stable 42,32 and 30 months, respectively, since the procedure. Figure 3 illustrates treatment planning in Case 1, and Fig. 4 illustrates visual fields before and 36 months after radiosurgery. This case is characterized by bilateral visual field impairment (left more than right) presumably related to the attraction and distortion of the optic chiasm caused by the tumor (Fig. 3). Bilateral improvement was noted after 6 months with full recovery visible after 12 months, and this status was maintained at 36 months. In terms of restoration of visual fields and acuity, similar results were also found in the other 2 patients, including the 1 with exophthalmos. This latter patient also experienced remission of the optociliary shunting approximately 1 year after the treatment in association with slightly reduced exophthalmos. The clinical outcome, including visual restoration, in this latter case was particularly interesting in view of the larger volume of the lesion $\left(6.4 \mathrm{~cm}^{3}\right.$, Table 1$)$.

The selection of this treatment protocol (total dose, dose per session, and number of sessions) was based on the knowledge of optic nerve tolerance to single-session radiosurgery ${ }^{12,14,16,19,26,28}$ and experience treating the anterior optic pathways and other cranial nerves with multisession radiosurgery. ${ }^{1,6,20}$ When using multisession radiosurgery, the the- 
TABLE 1

Summary of clinical characteristics and outcomes after radiosurgery

\begin{tabular}{ccccccc}
\hline \hline $\begin{array}{c}\text { Case } \\
\text { No. }\end{array}$ & $\begin{array}{c}\text { Age } \\
\text { (yrs), Sex }\end{array}$ & $\begin{array}{c}\text { Optic Nerve } \\
\text { Location }\end{array}$ & $\begin{array}{c}\text { Presenting } \\
\text { Symptom }\end{array}$ & $\begin{array}{c}\text { Visual } \\
\text { Acuity }\end{array}$ & $\begin{array}{c}\text { Volume } \\
\left(\mathrm{cm}^{3}\right)\end{array}$ & $\begin{array}{c}\text { Follow- } \\
\text { Up (mos) }\end{array}$ \\
\hline 1 & $42, \mathrm{~F}$ & lt & visual field loss & $20 / 30$ & 2.8 & $\begin{array}{c}\text { full restoration of visual fields \& acuity } \\
\text { full restoration of visual fields \& acuity }\end{array}$ \\
2 & $45, \mathrm{M}$ & $\mathrm{rt}$ & exophthalmos, visual field loss & $20 / 40$ & 6.4 & $\begin{array}{c}32 \\
\text { full restoration of visual fields \& acuity }\end{array}$ \\
3 & $54, \mathrm{~F}$ & rt & visual field \& acuity loss & $20 / 40$ & 1.1 & \\
\hline
\end{tabular}

rapeutic window is probably quite narrow; use of a more aggressive protocol (21 Gy delivered in 3 sessions of 7 Gy each) was associated with loss of vision in one patient. ${ }^{1}$ In contrast, the protocol used in our patients (20 Gy delivered in 4 sessions) induced fast resolution of visual symptoms and good preservation of vision after a mean follow-up duration of 37 months. This is the first report of multisession radiosurgery to treat ONSMs and, despite the small number of patients, it appears that this technique is quite promising; compared with conventional fractionated delivery, the duration of treatment in the present modality is much shortened while excellent accuracy, conformality, and homogeneity can be achieved due to image-guided frameless robotic delivery. Further studies expanding this preliminary experience are needed.

\section{Conclusions}

Optic nerve sheath meningiomas are slow-growing tumors causing progressive loss of vision. Optimal management is controversial, but it appears that early intervention using SFRT or radiosurgery can achieve growth control and stop the progression of visual deficits. Multisession image-guided robotic radiosurgery is a novel treatment modality that combines the highest degree of conformality and accuracy with the capacity to deliver treatment in sev-

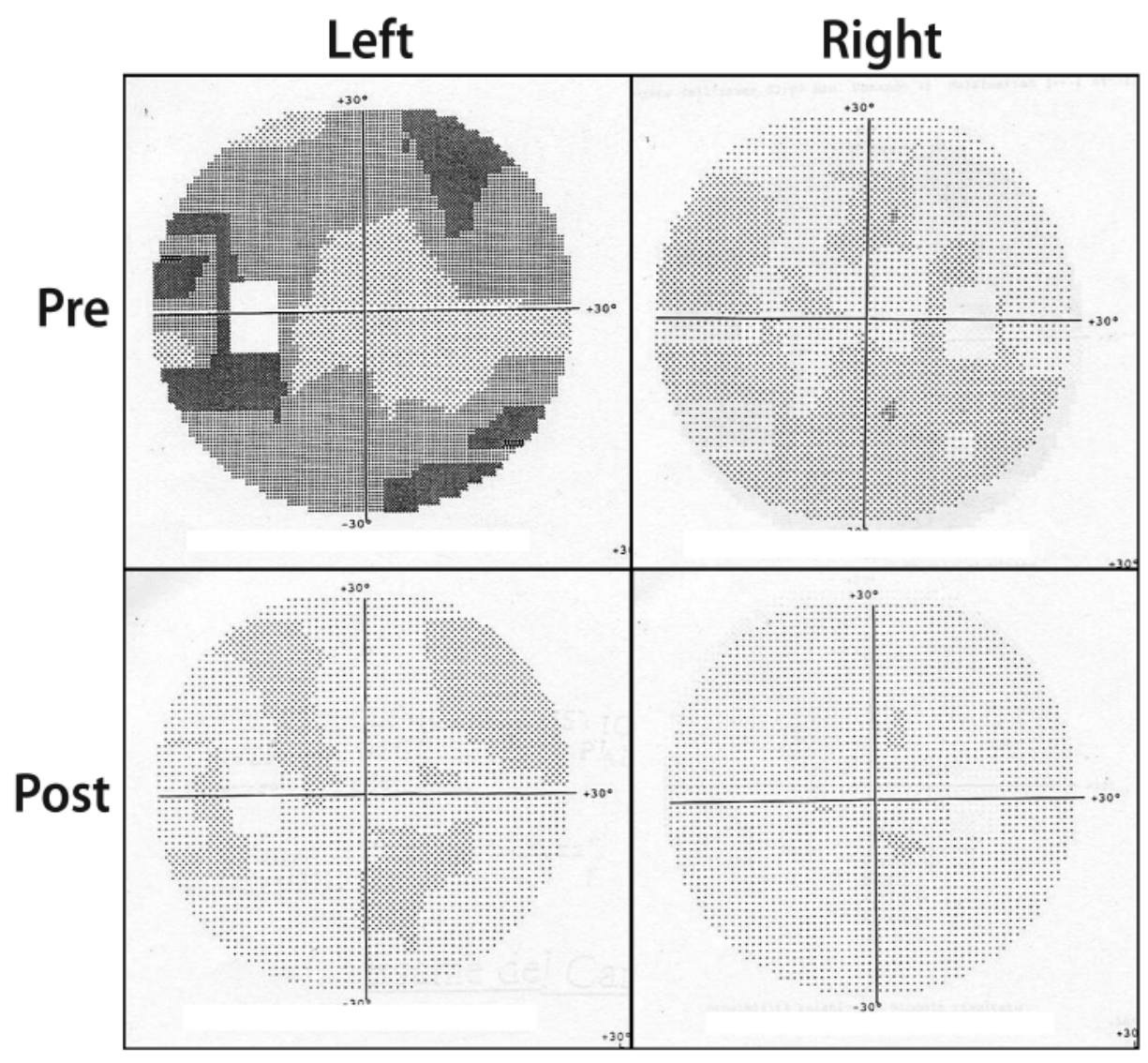

FIG. 4. Case 1. Left and right visual fields (left and right panels, respectively) obtained before (upper panels) and 36 months after (lower panels) treatment. Bilateral visual field deficits can be appreciated pretreatment, particularly affecting the left eye, where an ONSM was detected. Full restoration of visual fields was declared by an independent ophthalmologist. 
eral sessions and improve the tolerance of the optic nerve to the irradiation. Further studies are required to assess the value of multisession radiosurgery compared with singlesession radiosurgery and SFRT.

\section{Disclosure}

Dr. Romanelli has served in the past as a paid consultant for Accuray Incorporated. Dr. Wowra has served on Accuray's clinical advisory board.

\section{References}

1. Adler JR Jr, Gibbs IC, Puataweepong P, Chang SD: Visual field preservation after multisession cyberknife radiosurgery for perioptic lesions. Neurosurgery 59:244-254, 2006

2. Alper MG: Management of primary optic nerve meningiomas. Current status - therapy in controversy. J Clin Neuroophthalmol 1:101-117, 1981

3. Andrews DW, Faroozan R, Yang BP, Hudes RS, Werner-Wasik M, Kim SM, et al: Fractionated stereotactic radiotherapy for the treatment of optic nerve sheath meningiomas: preliminary observations of 33 optic nerves in 30 patients with historical comparison to observation with or without prior surgery. Neurosurgery 51:890-904, 2002

4. Becker G, Jeremic B, Pitz S, Buchgeister M, Wilhelm H, Schiefer $\mathrm{U}$, et al: Stereotactic fractionated radiotherapy in patients with optic nerve sheath meningioma. Int J Radiat Oncol Biol Phys 54:1422-1429, 2002

5. Boulos PT, Dumont AS, Mandell JW, Jane JA Sr: Meningiomas of the orbit: contemporary considerations. Neurosurg Focus 10(5): E5, 2001

6. Chang SD, Gibbs IC, Sakamoto GT, Lee E, Oyelese A, Adler JR Jr: Staged stereotactic irradiation for acoustic neuroma. Neurosurgery 56:1254-1263, 2005

7. Clark WC, Theofilos CS, Fleming JC: Primary optic nerve sheath meningiomas. Report of nine cases. J Neurosurg 70:37-40, 1989

8. Durkin SR, Roos D, Higgs B, Casson RJ, Selva D: Ophthalmic and adnexal complications of radiotherapy. Acta Ophthalmol Scand 85:240-250, 2007

9. Hintschich C, Rose G: Orbital tumors, in Tonn JC, Westphal M, Rutka JT, et al (eds): Neuro-Oncology of CNS Tumors. Berlin: Springer, 2006 pp 269-290

10. Klink DF, Miller NR, Williams J: Preservation of residual vision 2 years after stereotactic radiosurgery for a presumed optic nerve sheath meningioma. J Neuroophthalmol 18:117-120, 1998

11. Kwon Y, Bae JS, Kim JM, Lee DH, Kim SY, Ahn JS, et al: Visual changes after gamma knife surgery for optic nerve tumors. Report of three cases. J Neurosurg 102 Suppl:143-146, 2005

12. Leber KA, Berglöff J, Pendl G: Dose-response tolerance of the visual pathways and cranial nerves of the cavernous sinus to stereotactic radiosurgery. J Neurosurg 88:43-50, 1998

13. Lee AG, Woo SY, Miller NR, Safran AB, Grant WH, Butler EB: Improvement in visual function in an eye with a presumed optic nerve sheath meningioma after treatment with three-dimensional conformal radiation therapy. J Neuroophthalmol 16:247-251, 1996

14. Linskey ME, Flickinger JC, Lunsford LD: Cranial nerve length predicts the risk of delayed facial and trigeminal neuropathies after acoustic tumor stereotactic radiosurgery. Int J Radiat Oncol Biol Phys 25:227-233, 1993

15. Liu JK, Forman S, Moorthy CR, Benzil DL: Update on treatment modalities for optic nerve sheath meningiomas. Neurosurg Focus 14(5):E7, 2003
16. Morita A, Coffey RJ, Foote RL, Schiff D, Gorman D: Risk of injury to cranial nerves after gamma knife radiosurgery for skull base meningiomas: experience in 88 patients. J Neurosurg 90: 42-49, 1999

17. Moyer PD, Golnik KC, Breneman J: Treatment of optic nerve sheath meningioma with three-dimensional conformal radiation. Am J Ophthalmol 129:694-696, 2000

18. Narayan S, Cornblath WT, Sandler HM, Elner V, Hayman JA: Preliminary visual outcomes after three-dimensional conformal radiation therapy for optic nerve sheath meningioma. Int J Radiat Oncol Biol Phys 56:537-543, 2003

19. Ove R, Kelman S, Amin PP, Chin LS: Preservation of visual fields after peri-sellar gamma-knife radiosurgery. Int $\mathbf{J}$ Cancer 90: 343-350, 2000

20. Pham CJ, Chang SD, Gibbs IC, Jones P, Heilbrun MP, Adler JR Jr: Preliminary visual field preservation after staged CyberKnife radiosurgery for perioptic lesions. Neurosurgery 54:799-812, 2004

21. Pitz S, Becker G, Schiefer U, Wilhelm H, Jeremic B, Bamberg M, et al: Stereotactic fractionated irradiation of optic nerve sheath meningioma: a new treatment alternative. Br J Ophthalmol 86: 1265-1268, 2002

22. Romanelli P, Schaal DW, Adler JR: Image-guided radiosurgical ablation of intra- and extra-cranial lesions. Technol Cancer Res Treat 5:421-428, 2006

23. Romanelli P, Schweikard A, Schlaefer A, Adler JR: Computer aided robotic radiosurgery. Comput Aided Surg 11:161-174, 2006

24. Shimano H, Nagasawa S, Kawabata S, Ogawa R, Ohta T: Surgical strategy for meningioma extension into the optic canal. Neurol Med Chir (Tokyo) 40:447-452, 2000

25. Sinclair J, Marks MP, Levy RP, Adler JR Jr, Chang SD, Lopez JR, et al: Visual field preservation after curative multi-modality treatment of occipital lobe arteriovenous malformations. Neurosurgery 57:655-667, 2005

26. Stafford SL, Pollock BE, Leavitt JA, Foote RL, Brown PD, Link MJ, et al: A study on the radiation tolerance of the optic nerves and chiasm after stereotactic radiosurgery. Int J Radiat Oncol Biol Phys 55:1177-1181, 2003

27. Subramanian PS, Bressler NM, Miller NR: Radiation retinopathy after fractionated stereotactic radiotherapy for optic nerve sheath meningioma. Ophthalmology 111:565-567, 2004

28. Tishler RB, Loeffler JS, Lunsford LD, Duma C, Alexander E III, Kooy HM, et al: Tolerance of cranial nerves of the cavernous sinus to radiosurgery. Int J Radiat Oncol Biol Phys 27:215-221, 1993

29. Turbin RE, Thompson CR, Kennerdell JS, Cockerham KP, Kupersmith MJ: A long-term visual outcome comparison in patients with optic nerve sheath meningioma managed with observation, surgery, radiotherapy, or surgery and radiotherapy. Ophthalmology 109:890-900, 2002

30. van den Bergh AC, Dullaart RP, Hoving MA, Links TP, ter Weeme CA, Szabo BG, et al: Radiation optic neuropathy after external beam radiation therapy for acromegaly. Radiother Oncol 68:95-100, 2003

Manuscript submitted September 15, 2007.

Accepted October 17, 2007.

Address correspondence to: Pantaleo Romanelli, M.D., Department of Neurosurgery, IRCCS Neuromed, Via Atinense 18, Pozzilli, 86077, Italy. email: radiosurgery2000@yahoo.com. 